\title{
Oxaliplatin aggravates hepatic oxidative stress, inflammation and fibrosis in a non-alcoholic fatty liver disease mouse model
}

\author{
YULEI LU ${ }^{1}$, YOUZHI LIN ${ }^{2}$, XIAOQING HUANG ${ }^{1}$, SHENGMING WU ${ }^{3}$, JIAN WEI $^{1}$ and CHUN YANG $^{4}$ \\ ${ }^{1}$ Affiliated Tumor Hospital of Guangxi Medical University; Departments of ${ }^{2}$ Hepatobiliary Surgery, ${ }^{3}$ Pathology and \\ ${ }^{4}$ Experimental Pathology, Affiliated Tumor Hospital of Guangxi Medical University, Nanning, Guangxi 530021, P.R. China
}

Received November 5, 2018; Accepted March 27, 2019

DOI: $10.3892 /$ ijmm.2019.4154

\begin{abstract}
Oxaliplatin (OXA)-based chemotherapy is widely used in the treatment of gastrointestinal tumors; however, it is associated with chemotherapy-associated liver injury. Whether OXA induces liver injury and aggravates the already existing hepatic oxidative stress, inflammation and fibrosis in non-alcoholic fatty liver disease (NAFLD), and whether these effects can be alleviated by reduced glutathione (GSH) treatment, remains unclear. In the present study, OXA induced acute liver injury in NAFLD mice. Moreover, OXA increased the levels of reactive oxygen species (ROS) and malondialdehyde (MDA) and decreased the levels of superoxide dismutase and GSH peroxidase in the livers of NAFLD mice. OXA also induced the upregulation of hepatic inflammatory cytokines, such as tumor necrosis factor (TNF)- $\alpha$, interferon (IFN)- $\gamma$ and interleukin (IL)-17, in NAFLD mice. Furthermore, collagen fiber deposition in liver tissues was increased and the expression of transforming growth factor (TGF) $-\beta, \alpha$-smooth muscle actin (SMA) and tissue inhibitor of metallopeptidase (TIMP)-1 was upregulated in the livers of OXA-treated NAFLD mice. Treatment with exogenous GSH alleviated OXA-induced acute liver injury in NAFLD mice, and significantly reduced the levels of ROS, MDA and TNF- $\alpha$. However, GSH treatment did not inhibit collagen fiber deposition, although it reduced the levels of IFN- $\gamma$, IL-17, TGF- $\beta, \alpha$-SMA and TIMP-1 in the livers of OXA-treated NAFLD mice. In conclusion, OXA chemotherapy may induce acute liver injury and aggravate the existing hepatic oxidative stress, inflammation and fibrosis in NAFLD. Treatment of NAFLD mice with exogenous GSH alleviated OXA-induced liver injury, possibly by ameliorating OXA-aggravated hepatic oxidative stress and inflammation; it did not, however, attenuate OXA-aggravated liver fibrosis.
\end{abstract}

Correspondence to: Professor Chun Yang, Department of Experimental Pathology, Affiliated Tumor Hospital of Guangxi Medical University, 71 He Di Road, Nanning, Guangxi 530021, P.R. China

E-mail: cxl_yang@163.com

Key words: oxaliplatin, non-alcoholic fatty liver disease, oxidative stress, inflammation, fibrosis, glutathione

\section{Introduction}

Oxaliplatin (OXA), a third-generation platinum antitumor drug, is widely used for the treatment of gastrointestinal cancers, such as colorectal, gastric, liver and pancreatic cancer (1). As the first-line basic chemotherapeutic drug recommended by the National Comprehensive Cancer Network guidelines, OXA is currently used for the treatment of gastric and colorectal cancers $(1,2)$. However, despite its usefulness, OXA-based chemotherapy may cause chemotherapy-associated liver injury. Rubbia-Brandt et al (3) first reported that different degrees of hepatic sinus injuries occurred in $78 \%$ of colorectal cancer patients who received OXA-based chemotherapy. OXA-based chemotherapy-induced hepatic injury has been reported in as many as $19-52 \%$ of patients with various types of tumors (4). This injury may manifest as hepatic sinusoidal expansion, intrahepatic sinus platelet aggregation, hepatocyte atrophy and necrosis, hepatic steatosis, steatohepatitis, intrahepatic sinus hemorrhage and sinusoidal obstruction syndrome $(3,4)$.

At present, very little is known on the pathophysiological mechanisms that underlie OXA-induced liver injury. OXA has been confirmed to cause liver oxidative stress response through certain known mechanisms. Robinson et al (5) reported that oxidative stress-related genes (Mt1, HO1 and SOD3) were upregulated in the liver following OXA chemotherapy,indicating that oxidative stress plays an important role in OXA-induced liver injury. By generating reactive oxygen species (ROS), OXA causes a series of reactions, such as oxidative injury of normal hepatocyte mitochondria, as well as injury, falloff and local edema of sinusoidal endothelial cells, thereby causing chemotherapy-related liver injury (6). Our previous study also demonstrated that oxidative stress response plays an important role in OXA-induced acute liver injury (7).

Non-alcoholic fatty liver disease (NAFLD), which comprises a spectrum of liver diseases ranging from steatosis to non-alcoholic steatohepatitis and cirrhosis, is a common hepatic condition (8) that it is frequently associated with visceral obesity, dyslipidemia, insulin resistance and type-2 diabetes mellitus $(8,9)$. NAFLD is characterized by a low level of hepatic oxidative stress response, chronic inflammation and fibrosis (10). It was previously reported that, once chemotherapy-induced liver injury occurs in patients with NAFLD, it may cause adverse consequences, such as chemotherapy delay, dose reduction, or even cessation, as well as a 
higher risk of liver failure and death compared with patients without NAFLD (11). There is currently no standard of care in place for OXA-induced liver injury in patients with NAFLD. One of the hepatoprotective drugs used by clinicians is reduced glutathione (GSH). However, whether GSH treatment exerts protective effects against OXA-induced liver injury in NAFLD remains unclear.

OXA itself may cause hepatic oxidative stress response; therefore, whether OXA aggravates the already existing hepatic oxidative stress, inflammation and fibrosis in NAFLD remains unknown. The objectives of the present study were to investigate whether OXA chemotherapy affects the existing hepatic oxidative stress, inflammation and fibrosis in an NAFLD mouse model, and to investigate the protective action of GSH against OXA-induced liver injury in NAFLD.

\section{Materials and methods}

Ethics statement. All animal studies were performed according to the guidelines of the Chinese Council on Animal Care and were approved by the Affiliated Tumor Hospital of Guangxi Medical University (Nanning, China) Committees on Animal Experimentation.

Drugs and reagents. OXA for injection (cat. no. 13092615; Jiangsu Hengrui Medicine Co., Ltd.); GSH for injection (Chongqing Yaoyou Medicine Co., Ltd.); alanine aminotransferase (ALT) kit (HuiLi Biotech Co., Ltd.); aspartate aminotransferase (AST) kit, GSH kit, superoxide dismutase (SOD) kit, glutathione peroxidase (GSH-px) kit, malondialdehyde (MDA) kit and total protein quantification kit [bicinchoninic acid (BCA) method] (all from Jiangcheng Bioengineering Institute).

Animal experiments. BALB/cJ mice, aged 4-6 weeks, were purchased from Beijing Vital River Laboratory Animal Technology Co., Ltd. The mice were maintained in polypropylene cages ( $\mathrm{n}=6$ per cage) in an air-conditioned room $\left(25 \pm 1^{\circ} \mathrm{C}\right.$, relative humidity $50 \pm 20 \%, 12$-h light/dark cycle). After 1 week of acclimatization, the NAFLD mice were fed a high-fat diet (D12451, 45\% energy from fat; Research Diets, Inc.) for 12 weeks. At the end of the 12 weeks, the animals were administered $8 \mathrm{mg} / \mathrm{kg}$ OXA $(0.5 \mathrm{ml})$ via intraperitoneal (i.p.) injection for 3 days; the NAFLD control group was administered vehicle (5\% glucose, $0.5 \mathrm{ml}$ ) for 3 days. The drug regimen was based on previously published studies (7). Normal control mice were fed a standard chow diet for 12 weeks, and were then administered vehicle ( $5 \%$ glucose, $0.5 \mathrm{ml})$ for 3 days. The animals were anesthetized with ketamine/xylazine (100/15 mg/kg, i.m.), and blood and liver tissue were collected prior to sacrifice by exsanguination. Pieces of liver tissue were snap-frozen in liquid nitrogen, or were fixed in $10 \%$ neutral-buffered formalin. To assess the impact of GSH treatment, mice ( $n=10$ per group) were treated with OXA ( $8 \mathrm{mg} / \mathrm{kg}$, i.p.) for 3 days, and with GSH (400 mg/kg, i.p.) $30 \mathrm{~min}$ prior to the first OXA injection and once daily until 3 days after the final OXA dose. The mice were euthanized via deep anesthesia with isoflurane 3 days after the final dose of GSH was administered. Blood and liver samples were collected for further analysis. The experimental design is depicted in Fig. 1.
Histological examination. Liver tissues were fixed in $4 \%$ paraformaldehyde, embedded in paraffin and cut into $4-\mu \mathrm{m}$ sections. Histological assessment of the liver tissue sections was performed following hematoxylin and eosin (H\&E) and Masson's trichrome staining. The presence of liver injury was assessed by two specialist liver pathologists who were blinded to the grouping. Liver fibrosis was examined by Masson's trichrome staining. The morphometric assessment of liver fibrosis was performed using a fully automated Leica image processor with automated stage and Leica Quin software 2004 (Leica Microsystems $\mathrm{GmbH}$ ). The mean fibrotic area was calculated from 15-18 areas per liver section analyzed at a magnification of $\mathrm{x} 200$.

Flow cytometry and intracellular cytokine staining. Mononuclear cells (MNCs) were obtained from the mouse livers on the indicated days. Intracellular cytokine staining was performed as previously described (12). Briefly, MNCs were stimulated with phorbol myristate acetate (cat. no. P1585, 50 ng/ml; Sigma-Aldrich; Merck KGaA), Golgistop (cat. no. 554724, $2.0 \mu \mathrm{M}$; BD Biosciences), and ionomycin (cat. no. 407952, $1 \mu \mathrm{M}$; Calbiochem) for $5 \mathrm{~h}$. The cells were stained with phycoerythrin (PE)cy5.5-anti-CD3 (cat. no. 152312), and then fixed in $4 \%$ paraformaldehyde (cat. no.420801; both BioLegend). Next, the cells were permeabilized with phosphate-buffered saline (PBS; cat. no. SH30256.01B; HyClone) supplemented with $0.2 \%$ saponin and $0.05 \%$ sodium azide, and then stained with PE-anti-interleukin (IL)-4 (cat. no. 504104), PE-anti-IL-10 (cat. no. 505007), PE-anti-IL-17A (cat. no. 506903), PE-anti-interferon (IFN)- $\gamma$ (cat. no. 113603), PE-anti-tumor necrosis factor (TNF)- $\alpha$ (cat. no. 506305), or an isotype-matched, irrelevant control $\mathrm{Ab}$ (all from BioLegend). Stained cells were analyzed using a BD FACSAria flow cytometer (BD Biosciences).

Reverse transcription-quantitative polymerase chain reaction $(R T-P C R)$ analysis. Total RNA isolation and cDNA synthesis were conducted using standard methods. Total RNA was extracted using RNeasy Mini kits (Qiagen) and reverse-transcribed into cDNA using SuperScript III Reverse Transcriptase (Invitrogen; Thermo Fisher Scientific, Inc.). The gene-specific primers used are listed in Supplementary Table S1. RT-qPCR was performed with ABI PRISM 7500 Real-Time PCR System (Applied Biosystems; Thermo Fisher Scientific, Inc.) with $1 X$ SYBR-Green Universal PCR Mastermix (Takara Bio, Inc.). Transcript levels were calculated according to the $2^{-\Delta \Delta \mathrm{Cq}}$ method, normalized to the expression of GAPDH, and expressed as fold change compared with the control. All samples were run in duplicate to ensure amplification integrity.

Analysis of oxidative stress parameters. Proteins were extracted from whole liver tissues using RIPA buffer and quantified using the Bradford assay (Nanjing Jiangcheng Bioengineering Institute). The SOD, MDA and GSH-Px content of liver tissues was determined using the kits obtained from Nanjing Jiangcheng Bioengineering Institute, according to the manufacturer's protocols. To estimate hepatic ROS level, liver tissues were harvested and immediately homogenized in PBS using a Teflon homogenizer (Tissue-Tearor; BioSpec Products Inc.). Briefly, $50 \mu 1$ liver homogenate was mixed 


\section{Normal control $(\mathrm{CN})$ : standard chow diet 12 weeks, then inject $5 \%$ glucose $(0.5 \mathrm{ml} / \mathrm{day} / 3$ days $)$ \\ 2. NAFLD control (NA): high-fat diet 12 weeks, then inject $5 \%$ glucose $(0.5 \mathrm{ml} / \mathrm{day} / 3$ days $)$ \\ 3 OXA (NA+OXA): high-fat diet 12 weeks, then inject OXA ( $8 \mathrm{mg} / \mathrm{kg} / \mathrm{day} / 3$ days) \\ 4. GSH control (NA+GSH): high-fat diet 12 weeks, then inject $\mathrm{GSH}(400 \mathrm{mg} / \mathrm{kg} /$ day/6 days) \\ 4. OXA+GSH (NA+OXA+GSH): high-fat diet 12 weeks, then inject OXA ( $8 \mathrm{mg} / \mathrm{kg} / \mathrm{day} / 3$ days) plus GSH (400 mg/kg/day/6 days)}

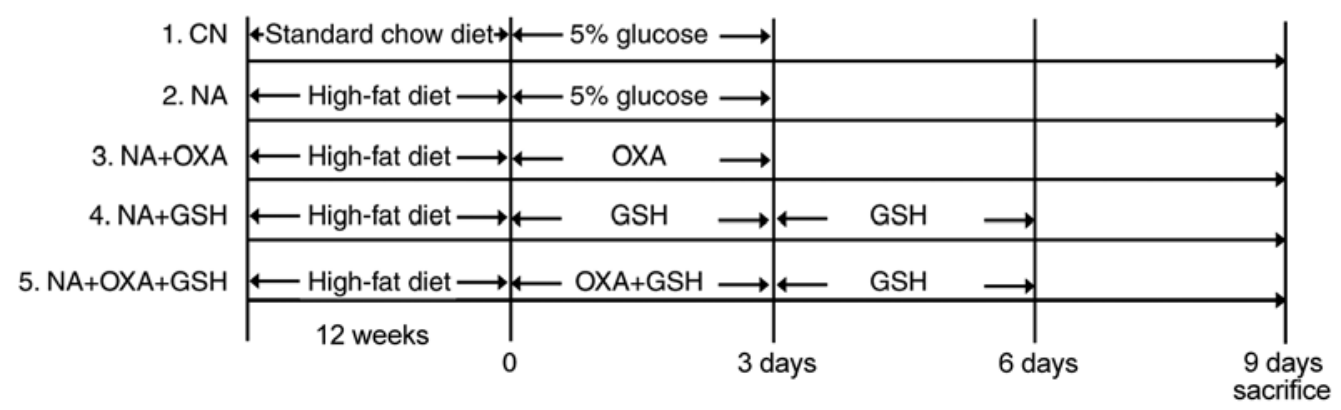

Figure 1. Experimental design. Five groups of mice were used in the present study. Standard chow diet and high-fat diet were administered orally; OXA, $5 \%$ glucose and GSH were injected intraperitoneally. OXA, oxaliplatin; GSH, reduced glutathione.

with $4.85 \mathrm{ml}$ of $100 \mathrm{mmol} / \mathrm{l}$ potassium phosphate buffer (cat. no. 700621-5; Cayman Chemical) and incubated with 2',7'-dichlorofluorescin (DCF) diacetate (Sigma-Aldrich; Merck KGaA) in methanol at a final concentration of $5 \mu \mathrm{mol} / 1$ for $15 \mathrm{~min}$ at $37^{\circ} \mathrm{C}$. The hepatic ROS level was determined as the amount of 2',7'-dichlorofluorescein (DCF) quantified from a DCF standard curve. The BCA protein assay kit (Thermo Fisher Scientific, Inc.) was used to measure protein concentration. The fluorescence of DCF was measured with a Varioskan spectrophotometer (Thermo Fisher Scientific, Inc.) at excitation/emission wavelengths $480 / 530 \mathrm{~nm}$.

Statistical analysis. The results are expressed as means \pm standard deviation. Cumulative survival time was calculated using the Kaplan-Meier method and was analyzed by the log-rank test. Other datasets were evaluated by ANOVA with a post hoc Tukey's test for multiple comparisons. All statistical analyses were performed using SPSS 10 (SPSS Inc.) and $\mathrm{P}<0.05$ was considered to indicate statistically significant differences.

\section{Results}

OXA chemotherapy induces acute liver injury in NAFLD mice. Balb/c mice were first fed a high-fat diet for 12 weeks to establish NAFLD and were then treated with OXA (i.p.) once per day for 3 days (Fig. 1). Survival curve analysis revealed that death occurred 2 days after the administration of the final dose of OXA, and the survival rate in this group was $50 \%$ (5/10; Fig. 2A). The weight of the OXA-treated mice was significantly lower compared with that of the NAFLD control group (Fig. 2B; $\mathrm{P}<0.05$ ). To evaluate OXA-induced liver injury in NAFLD mice, the serum AST and alanine aminotransferase (ALT) levels were assessed. Compared with the control mice, the OXA-treated mice exhibited significantly elevated serum ALT and AST levels following OXA treatment (Fig. 2C; $\mathrm{P}<0.05)$. Coinciding with the trend of serum ALT and AST levels, more severe inflammatory cell infiltration, hepatocyte ballooning and necrosis were observed in the OXA-treated mice (Fig. 2D). Moreover, the lipid vacuoles in the OXA group were more abundant and larger compared with those observed in the control group (Fig. 2D). Additionally, no significant hepatic sinus expansion was observed in the liver tissues of the OXA group mice. These results indicated that OXA chemotherapy causes acute liver injury in NAFLD mice.

OXA chemotherapy aggravates intrahepatic oxidative stress response in NAFLD mice. High-fat diet may induce oxidative stress in the NAFLD liver (13). The findings of the present study indicated that OXA-induced liver injury is associated with oxidative stress. To observe the effects of OXA on intrahepatic oxidative stress in NAFLD mice, the levels of hepatic MDA, ROS, SOD and GSH-Px were measured as an indication of the redox status of NAFLD mice following OXA treatment. The levels of the oxidative indicators MDA (Fig. 3A) and ROS (Fig. 3B) in the liver tissues of the NAFLD mice were found to be significantly increased following OXA injection $(\mathrm{P}<0.05)$. By contrast, the levels of the antioxidative indices SOD (Fig. 3C) and GSH-Px (Fig. 3D) in the liver tissues of NAFLD mice decreased following OXA treatment. The liver tissues exhibited marked alterations in the expression of redox status indicators following OXA injection, indicating that OXA aggravated the intrahepatic oxidative stress response in NAFLD mice.

OXA aggravates intrahepatic inflammatory response in NAFLD mice. Subclinical inflammation has also been reported as one of the basic changes that occur in NAFLD (8). In order to investigate the effect of OXA chemotherapy on intrahepatic inflammation in NAFLD mice, flow cytometry was performed to detect the expression of inflammatory cytokines in intrahepatic $\mathrm{T}$ lymphocytes in NAFLD mice following OXA chemotherapy. The percentage of TNF- $\alpha-$, IFN- $\gamma-$ and IL-17-producing $\mathrm{T}$ cells significantly increased 6 days after the administration of the first dose of OXA $(\mathrm{P}<0.05)$, whereas no significant change in the percentage of IL-6-producing T cells was observed (Fig. 4A and B, P>0.05). Similarly, the TNF- $\alpha$, IFN- $\gamma$ and IL-17 mRNA levels in the OXA group 

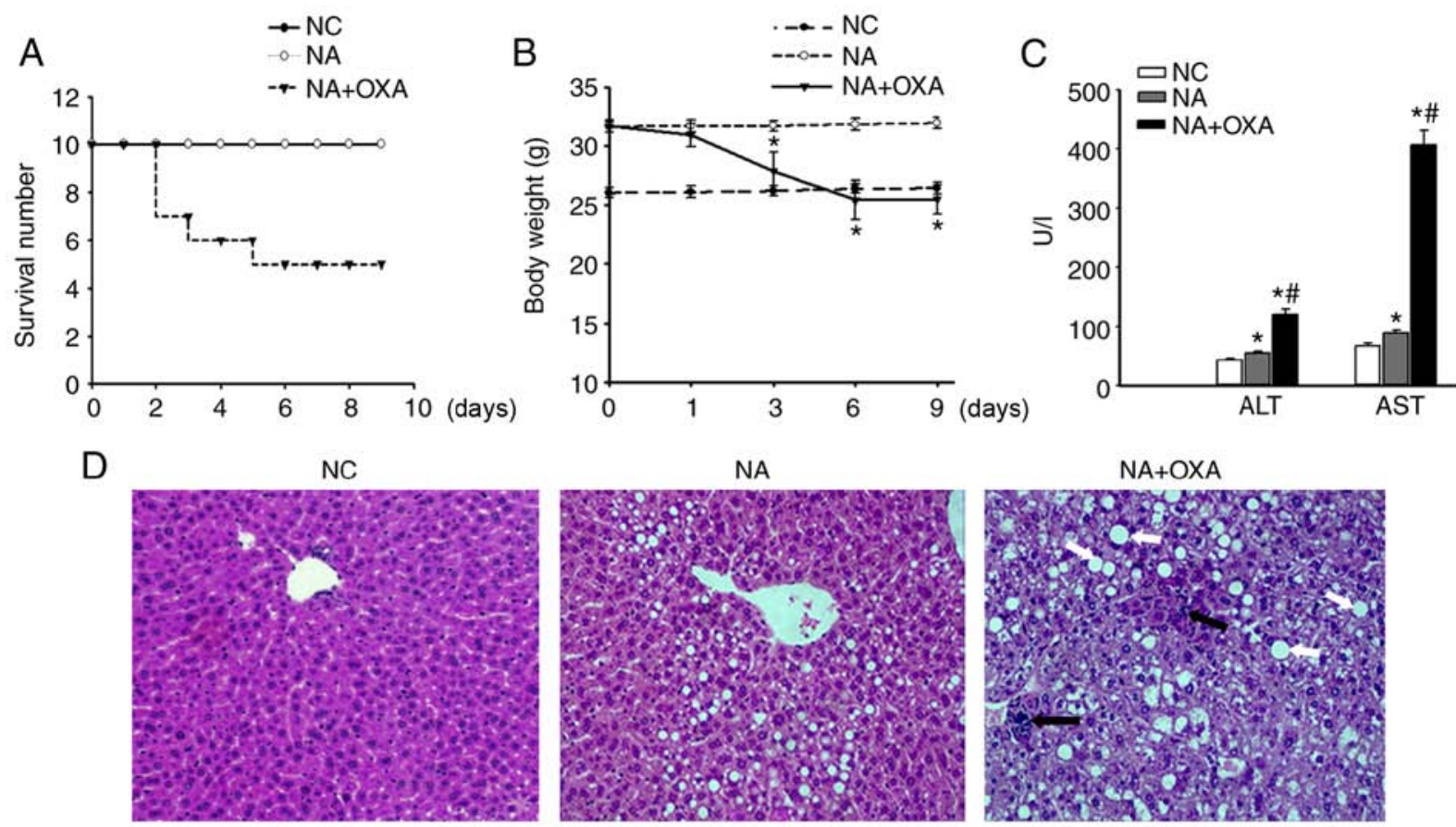

Figure 2. OXA-induced acute liver injury in NAFLD mice. (A) Survival rates of the three groups. No mice died in either the NC or the NA groups, and the survival rate across the 9 days was the same in both groups. (B) Changes in body weight were observed in the three study groups. (C) ALT and AST serum levels 3 days after the administration of the final dose of OXA. The results are presented as the mean \pm standard deviation of five mice in each group. ${ }^{*} \mathrm{P}<0.05$ compared with the $\mathrm{NC}$ group; ${ }^{~} \mathrm{P}<0.05$ compared with the NA group. (D) Histopathological examination of liver tissues of the control groups and 3 days after the administration of the final dose of OXA (H\&E staining; original magnification, x100). Black arrows indicate inflammatory infiltration, and white arrows indicate large lipid vacuoles. OXA, oxaliplatin; NAFLD, non-alcoholic fatty liver disease; ALT, alanine aminotransferase; AST, aspartate aminotransferase; NC, normal control; NA, NAFLD control; H\&E, hematoxylin and eosin.

A

A

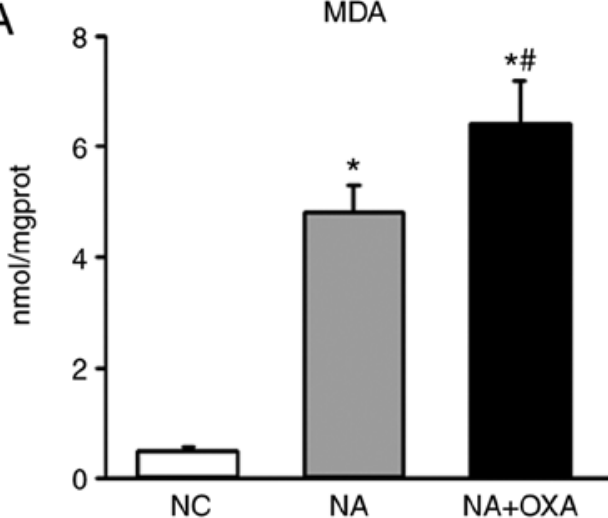

C

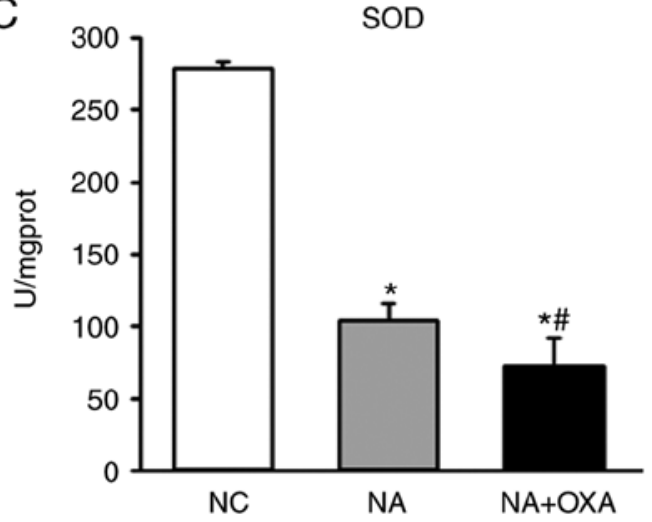

B
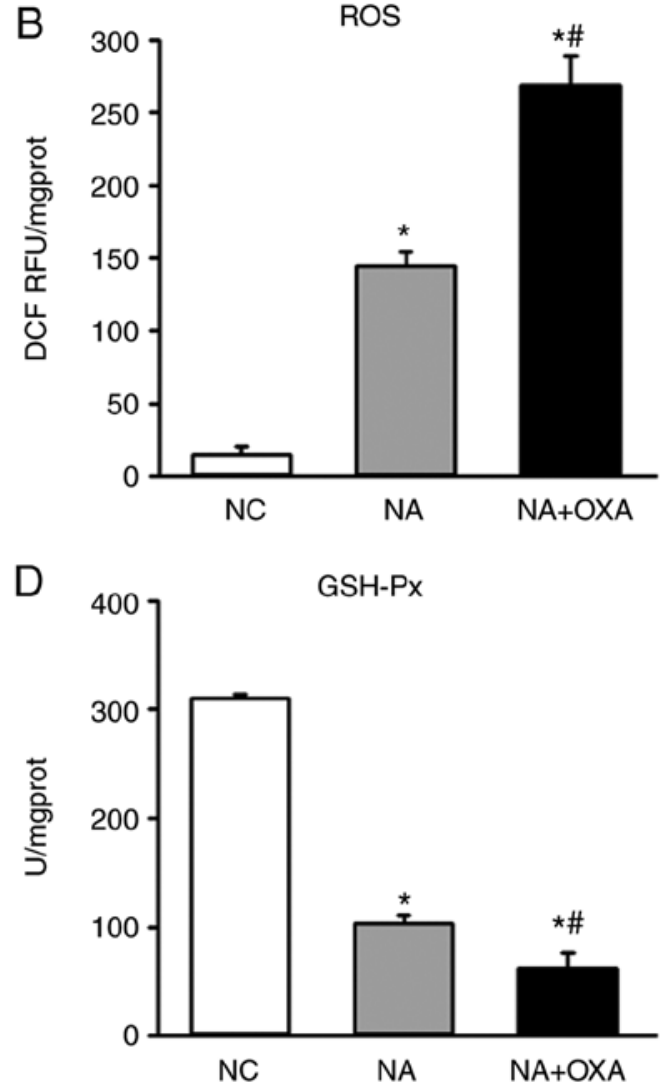

Figure 3. Effects of OXA on oxidative stress. Levels of (A) MDA, (B) ROS, (C) SOD and (D) GSH-Px in the liver tissues of each group of mice 3 days after the administration of the final dose of OXA. Results are presented as the mean \pm standard deviation of five mice in each group. "P $<0.05$ compared with the NC group; ${ }^{\#} \mathrm{P}<0.05$ compared with the NA group. OXA, oxaliplatin; MDA, malondialdehyde; ROS, reactive oxygen species; SOD, superoxide dismutase; GSH-Px, glutathione peroxidase; NC, normal control; NA, NAFLD control. 

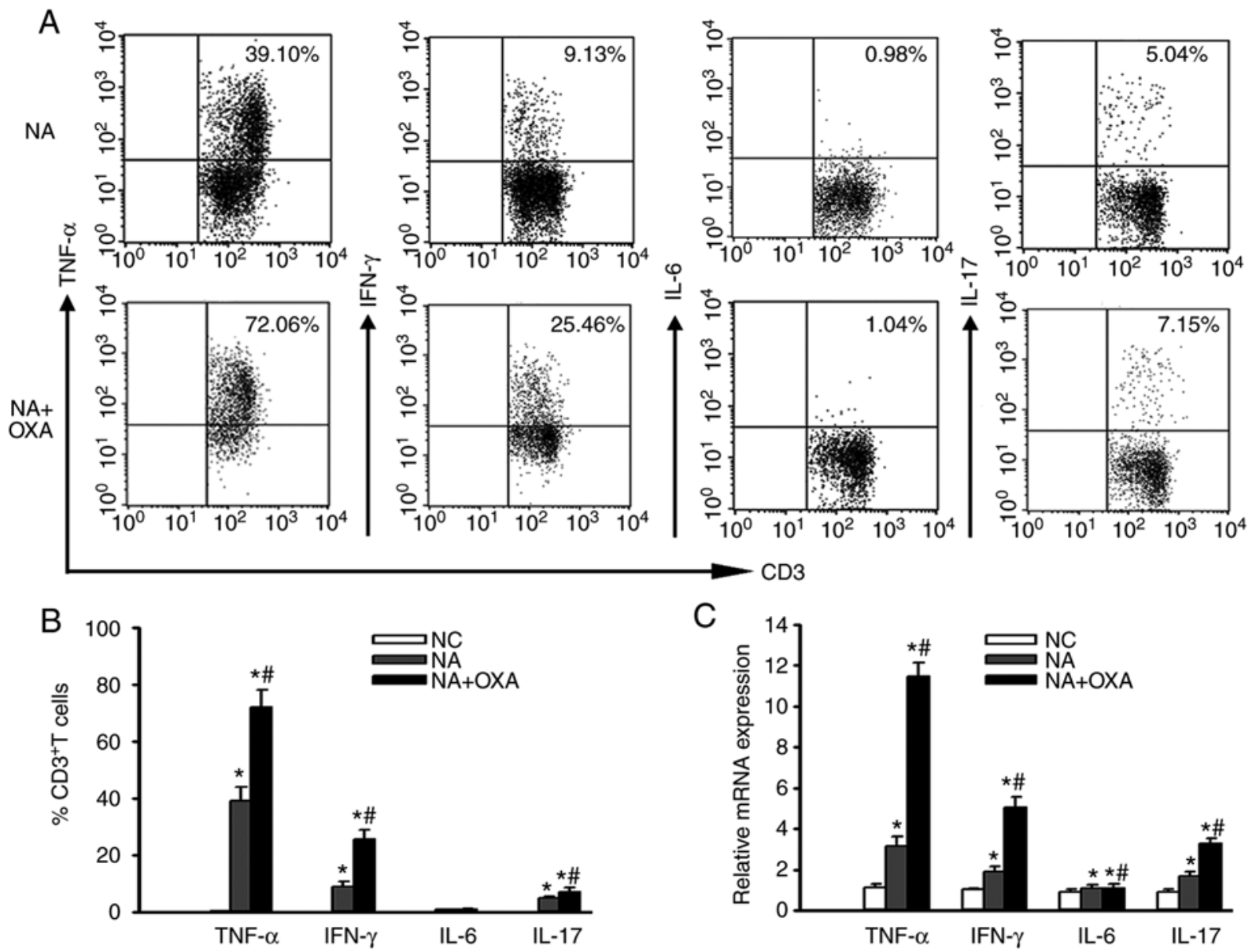

Figure 4. Effects of OXA on inflammatory cytokine expression. (A) Representative staining of intracellular TNF- $\alpha$, IFN- $\gamma$, IL-6 and IL-17 in hepatic CD $3^{+}$ T cells from the NA + OXA group 3 days after the administration of the final dose of OXA. (B) Percentages of CD $3^{+}$T cells expressing TNF- $\alpha$, IFN- $\gamma$, IL- 6 and IL-17 from the livers of each group of mice 3 days after the administration of the final dose of OXA. (C) RT-PCR was employed to investigate the mRNA levels of TNF- $\alpha$, IFN- $\gamma$, IL- 6 and IL-17 in the liver tissues of each group of mice 3 days after the administration of the final dose of OXA. Data represent the mean \pm standard deviation of five mice in each group. ${ }^{*} \mathrm{P}<0.05$ compared with the $\mathrm{NC}$ group; ${ }^{*} \mathrm{P}<0.05$ compared with the NA group. OXA, oxaliplatin; TNF, tumor necrosis factor; IFN, interferon; IL, interleukin; RT-PCR, reverse transcription-polymerase chain reaction; NC, normal control; NA, NAFLD control.

were significantly higher compared with those in the control group $(\mathrm{P}<0.05$; Fig. $4 \mathrm{C})$. These results indicated that OXA may aggravate intrahepatic inflammation in NAFLD mice.

OXA chemotherapy promotes hepatic fibrosis in NAFLD mice. Next, the effect of OXA on hepatic fibrosis in NAFLD mice was investigated. Hepatopathological analysis did not reveal distinct liver fibrosis 6 days after administration of the first dose of OXA (Fig. 2D). Masson's trichrome staining revealed small amounts of collagen fiber deposits in the liver tissues of NAFLD mice prior to OXA treatment, but increased collagen fiber deposition in the liver 6 days after administration of the first dose of OXA; these changes were more prominent 10 days after OXA treatment, as distinct circumferential collagen fiber deposition was observed in hepatocytes (Fig. 5A and B). Consistent with these findings, the levels of the liver fibrosis index transforming growth factor (TGF) $\beta$ significantly increased 6 days after administration of the first dose of OXA (Fig. 5C). There was also a modest increase in $\alpha$-smooth muscle actin (SMA) and tissue inhibitor of metallopeptidase (TIMP)-1 expression 6 days after administration of the first dose of OXA, but these differences did not reach statistical significance (Fig. 5D and E). However, liver fibrosis was aggravated, which was confirmed by a significant increase in the mRNA levels of TGF- $\beta$ (4.85-fold; P<0.01), $\alpha$-SMA (2.23-fold; $\mathrm{P}<0.05)$ and TIMP-1 (2.64-fold; $\mathrm{P}<0.01) 10$ days after OXA treatment (Fig. 5C-E). These results confirmed that OXA promotes hepatic fibrosis in NAFLD mice.

GSH attenuates OXA-induced acute liver injury in NAFLD mice. Our previous research demonstrated that treatment with GSH attenuates OXA-induced acute liver injury in normal mice (7). In the present study, to test whether GSH exerts a protective effect against OXA-induced acute liver injury in NAFLD mice, OXA-treated NAFLD mice underwent GSH treatment 30 min prior to each OXA injection for 3 days, as well as for 3 consecutive days after the final dose of OXA (NA + OXA + GSH group) (Fig. 1). Treatment with GSH did not improve the survival rate of OXA-treated NAFLD mice (Fig. 6A). However, hepatopathological analysis indicated severe swelling and necrosis of hepatocytes, destruction of the hepatic architecture and inflammatory cell infiltration in OXA-treated NAFLD mice, which was attenuated by GSH treatment (Fig. 6B). Additionally, there were fewer and smaller lipid vacuoles in the hepatocytes of the NA + OXA + GSH group (Fig. 6B). Consistent with histological findings, 

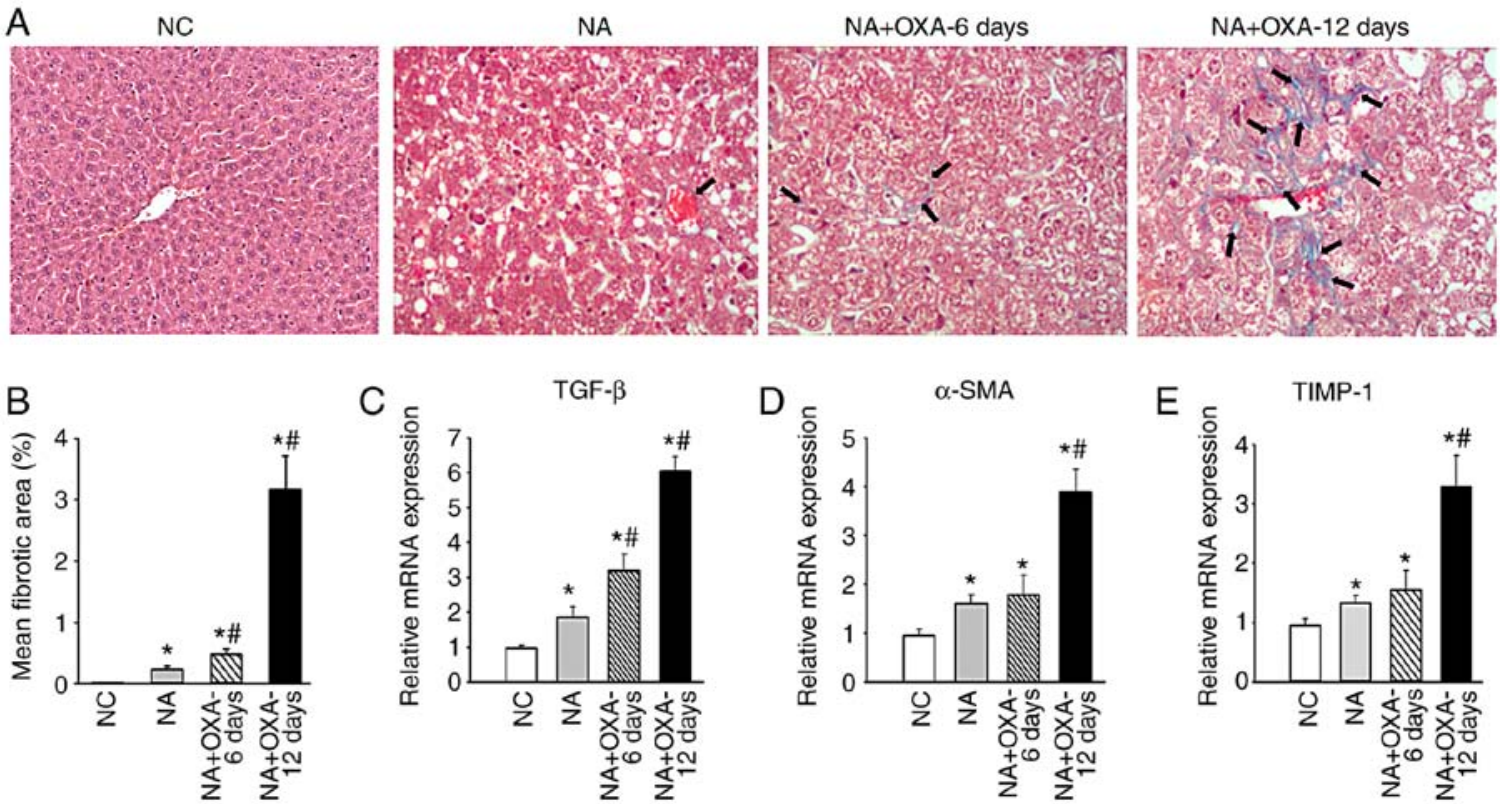

Figure 5. Effects of OXA on hepatic fibrosis in NAFLD mice. (A) Masson's trichrome staining on the liver specimens in groups NC, NA and NA + OXA (at 6 and 12 days after the administration of the first dose of OXA). Black arrows indicate areas of collagen deposition. (B) Morphometric analysis of Masson's trichrome staining of the groups in (A). (C-E) mRNA levels of (C) TGF- $\beta$, (D) $\alpha$-SMA and (E) TIMP-1 in liver tissues analyzed by RT-PCR. Results are presented as the means \pm standard deviation from five mice in each group. ${ }^{*} \mathrm{P}<0.05$ compared with the NC group; ${ }^{\sharp} \mathrm{P}<0.05$ compared with the NA group. OXA, oxaliplatin; NAFLD, non-alcoholic fatty liver disease; NC, negative control; NA, NAFLD control; TGF, transforming growth factor; SMA, smooth muscle actin; TIMP, tissue inhibitor of metallopeptidase; RT-PCR, reverse transcription-quantitative polymerase chain reaction.

treatment with GSH inhibited OXA-induced increases in the serum levels of ALT (Fig. 6C; P<0.05) and AST (Fig. 6C; $\mathrm{P}<0.05)$. GSH treatment did not alter the serum levels of albumin, coagulation times or total proteins (data not shown). These results indicated that GSH attenuates OXA-induced acute liver injury in NAFLD mice.

GSH attenuates OXA-aggravated oxidative stress and hepatic inflammation in NAFLD mice. To test whether GSH exerts a protective effect against the oxidative stress aggravated by OXA in NAFLD mice, OXA-administered NAFLD mice underwent GSH treatment. The analysis of oxidative stress indices indicated that the MDA (Fig. 7A; $\mathrm{P}<0.05$ ) and ROS (Fig. 7B; $\mathrm{P}<0.05$ ) levels in the liver tissues of the $\mathrm{NA}+\mathrm{OXA}+\mathrm{GSH}$ group were significantly lower compared with those in non-GSH-treated mice (NA + OXA group) following OXA injection. The levels of SOD (Fig. 7C; $\mathrm{P}<0.05$ ) and GSH-Px (Fig. 7D; $\mathrm{P}<0.05)$ in the liver tissues of the $\mathrm{NA}+\mathrm{OXA}+\mathrm{GSH}$ group were significantly higher compared with those in the NA + OXA group. These results indicated that GSH treatment may mitigate the OXA-aggravated intrahepatic oxidative stress response in NAFLD mice following OXA chemotherapy.

The findings of the present study confirmed that OXA can increase the expression of the inflammatory cytokines TNF- $\alpha$, IFN- $\gamma$ and IL-17 in the livers of NAFLD mice. Subsequently, the expression of these cytokines was detected in liver tissues following GSH treatment. It was observed that treatment with GSH reduced the percentage of hepatic TNF- $\alpha^{+} \mathrm{T}$ cells in the NA + OXA + GSH group (Fig. 7E; P<0.05), whereas no significant difference in the proportion of hepatic IFN- $\gamma^{+}$and IL-17 ${ }^{+} \mathrm{T}$ cells was observed compared with the NA + OXA group (Fig. 7F and G; P>0.05). Similarly, treatment with GSH resulted in lower TNF- $\alpha$ mRNA levels in the liver tissues of the $\mathrm{NA}+\mathrm{OXA}+\mathrm{GSH}$ group, whereas no significant differences in the IFN- $\gamma$, IL- 6 and IL-17 mRNA levels were observed compared with those in the NA + OXA group (Fig. $7 \mathrm{H}$ ). These results indicated that GSH treatment suppressed the OXA-aggravated inflammation by reducing the expression of the inflammatory cytokine TNF- $\alpha$, but did not affect the expression of IFN- $\gamma$, IL- 6 or IL-17.

GSH does not attenuate OXA-aggravated liver fibrosis in NAFLD mice. Whether GSH treatment attenuates OXA-aggravated liver fibrosis in NAFLD mice was next investigated. GSH treatment did not decrease the mean fibrotic area in the liver tissues of the NA + OXA + GSH group compared with the NA + OXA group (Fig. 8A and B; P>0.05). Additionally, treatment with GSH resulted in a mild decrease in the levels of $\alpha$-SMA, TGF- $\beta$ and TIMP- 1 compared with the $\mathrm{NA}+\mathrm{OXA}$ group, but the differences did not reach statistical significance (Fig. 8C-E; P>0.05). These results indicated that GSH treatment did not mitigate hepatic fibrosis in NAFLD mice after OXA chemotherapy.

\section{Discussion}

There are currently several challenges associated with the clinical studies attempting to investigate the effect of OXA chemotherapy on the progression of NAFLD. First, non-invasive methods, such as ultrasound and magnetic resonance elastography, may achieve an accurate diagnosis of moderate-to-severe steatosis (defined as histological degree $\geq 30 \%$ ) (14); however, they have limited sensitivity in mild steatosis (defined as histological degree 5-30\%) and may result in the misdiagnosis of patients as healthy controls (14). Liver biopsy, the current 

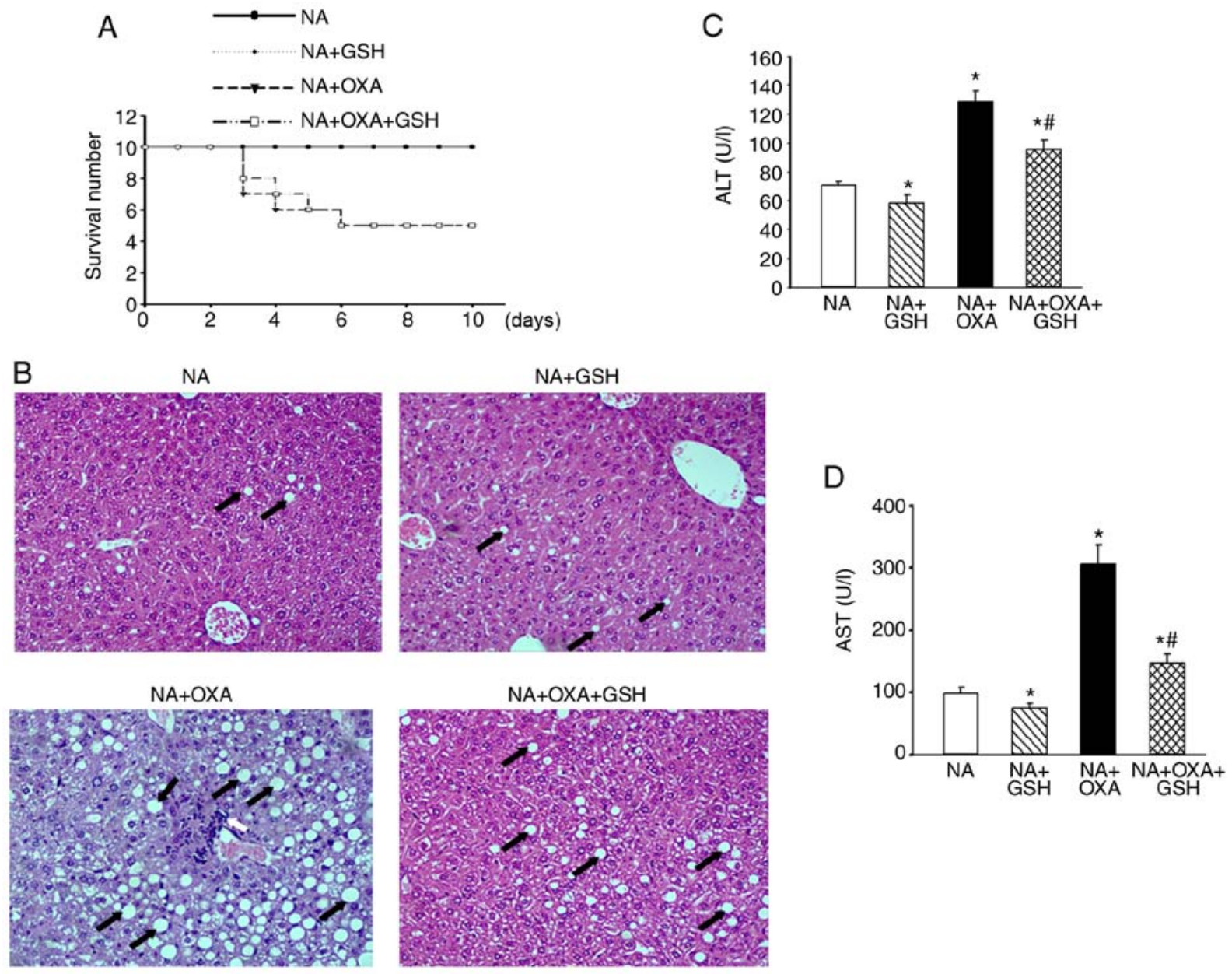

Figure 6. Treatment with GSH attenuates OXA-induced acute liver injury in NAFLD mice. NAFLD mice were randomly classified into four groups: i) NAFLD mice treated with OXA for 3 days (NA + OXA); ii) NAFLD mice treated with OXA for 3 days and with GSH every day from the first day of OXA administration until the end of the experiment (NA + OXA + GSH); iii) NAFLD mice treated with GSH every day (NA + GSH); and iv) NAFLD mice that received $5 \%$ glucose for 3 days, serving as control (NA). (A) The survival rates of the four groups were assessed. No mice died in either the NA or NA + GSH groups, and the survival rate across the 10 days was the same in both group. (B) Liver histopathology was examined in each group 6 days after the final dose of OXA (H\&E staining, original magnification, x100). White arrow indicates inflammatory infiltration, and black arrows indicate lipid vacuoles. The serum (C) ALT and (D) AST levels of each group were evaluated 6 days after the final dose of OXA. The results are presented as the mean \pm standard deviation from five mice in each group. "P<0.05 compared with the NA group; ${ }^{*} \mathrm{P}<0.05$ compared with the NA + OXA group. GSH, reduced glutathione; OXA, oxaliplatin; NAFLD, non-alcoholic fatty liver disease; H\&E, hematoxylin and eosin; ALT, alanine aminotransferase; AST, aspartate aminotransferase; NA, NAFLD control.

gold standard for diagnosing NAFLD, is costly, invasive and potentially risky (14). Therefore, liver biopsy or non-invasive methods may be unsuitable for screening patients to assess the progression of NAFLD following OXA chemotherapy, or for patient follow-up after therapeutic intervention. Animal models can overcome the shortcomings of the abovementioned clinical studies for investigating the effect of OXA chemotherapy on the progression of NAFLD. We previously established an animal model with OXA-induced acute liver injury in normal healthy mice (7). In the present study, OXA was used to induce acute liver injury in mice with high-fat diet-induced NAFLD. More severe lipid accumulation, inflammatory cell infiltration and hepatocyte necrosis were observed in the NAFLD mice following OXA chemotherapy. Moreover, the levels of serum ALT and AST were consistent with the histological findings. These results demonstrated that OXA may induce acute liver injury in NAFLD mice. To the best of our knowledge, this is the first report of a reproducible experimental NAFLD model with OXA-induced acute liver injury, which may be used to explore in detail the pathogenesis of this condition.
It was previously indicated that long-term high-fat diet can significantly increase free radical production and alter the body's redox status, ultimately resulting in chronic oxidative stress in liver tissues (13). The present study demonstrated that high-fat diet also induced chronic oxidative stress in the liver tissues of NAFLD mice. This oxidative stress was further exacerbated by OXA chemotherapy, as confirmed by a significant increase in the levels of MDA and ROS in the liver tissues of NAFLD mice, as well as a significant decrease in the activity of the antioxidant enzymes SOD and GSH-Px. Oxidative stress may lead to hepatocellular injury through several mechanisms, including lipid peroxidation, which can directly promote cell necrosis and activate the apoptotic Fas-ligand pathway (15). Oxidative stress occurs when ROS are produced at levels exceeding those capable of being sequestered by normal cellular antioxidant defenses $(13,15)$. Excessive amounts of ROS may exert direct deleterious effects on cells through lipid peroxidation, protein degradation and DNA damage $(15,16)$. In line with the generation of high amounts of ROS, the livers of NAFLD mice treated with OXA 
A
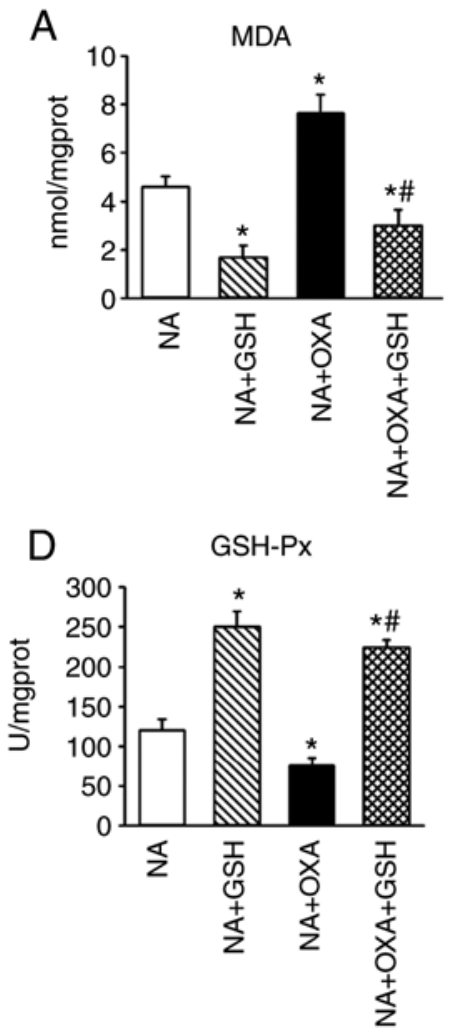

G

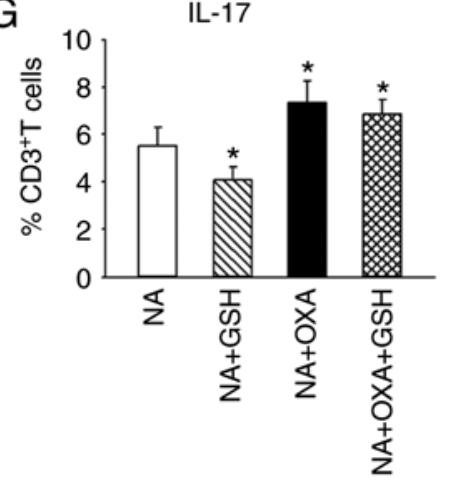

B

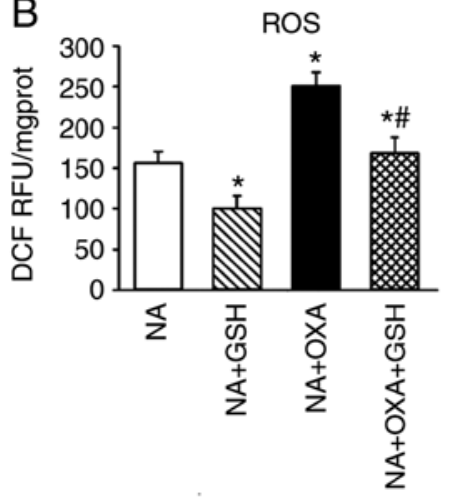

E

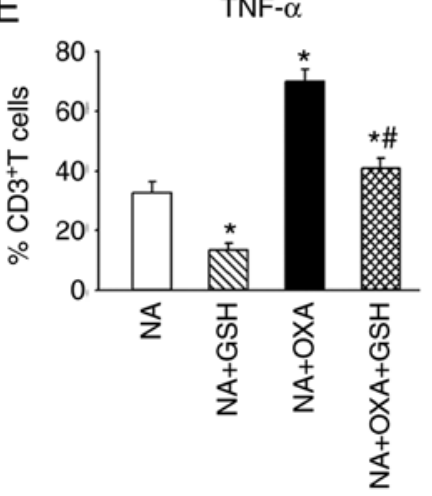

C

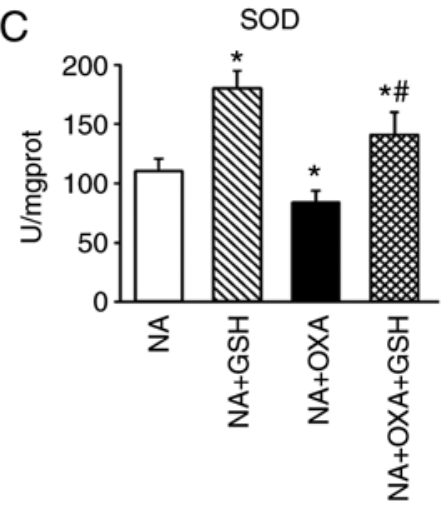

$\mathrm{F}$

$\mathrm{F} \quad \mathrm{IFN}-\gamma$

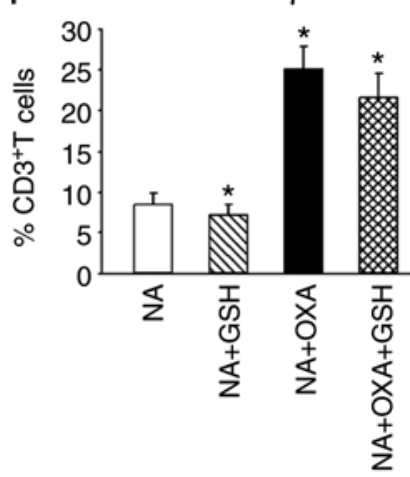

$\square \mathrm{NA}$

$\square \mathrm{NA}+\mathrm{GSH}$

NA+OXA

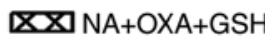
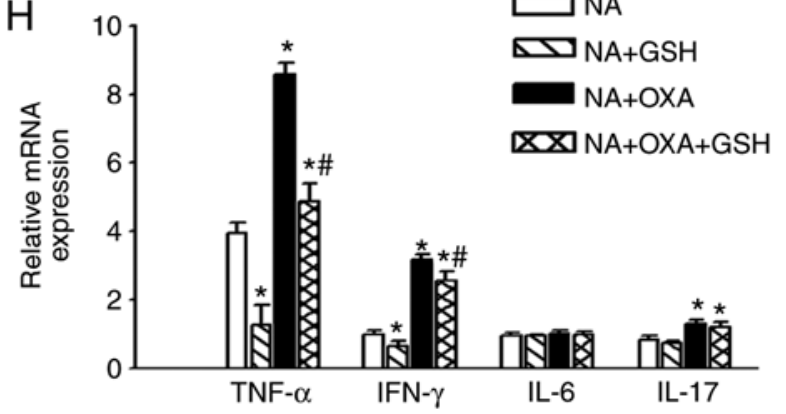

Figure 7. GSH attenuates OXA-aggravated hepatic oxidative stress and inflammation in NAFLD mice. (A-D) RT-PCR was employed to investigate the mRNA levels of (A) MDA, (B) ROS, (C) SOD and (D) GSH-Px in the liver tissues of each group 9 days after the first dose of OXA. (E-G) Flow cytometry detection of the intracellular cytokines (E) TNF- $\alpha,(\mathrm{F})$ IFN- $\gamma$ and (G) IL-17 was performed on CD3 ${ }^{+} \mathrm{T}$ cells from each group 9 days after the first dose of OXA. (H) mRNA levels of TNF- $\alpha$, IFN- $\gamma$ and IL-17 in the liver tissues of each group 3 days after the final dose of OXA. The results are presented as the mean \pm standard deviation from five mice in each group. " $\mathrm{P}<0.05$ compared with the NA group; ${ }^{*} \mathrm{P}<0.05$ compared with the NA + OXA group. GSH, reduced glutathione; OXA, oxaliplatin; NAFLD, non-alcoholic fatty liver disease; RT-PCR, reverse transcription-polymerase chain reaction; MDA, malondialdehyde; ROS, reactive oxygen species; SOD, superoxide dismutase; GSH-Px, glutathione peroxidase; TNF, tumor necrosis factor; IFN, interferon; IL, interleukin; NA, NAFLD control.

exhibited severe pathological changes, as demonstrated by a higher degree of inflammatory cell infiltration, hepatocyte necrosis and increased serum transferase activity. This indicates that OXA can amplify the existing oxidative stress in NAFLD, and induce oxidative liver injury.

Hepatic inflammation is considered to be the main driver of hepatic tissue damage, triggering the progression from NAFLD to severe fibrogenesis and, ultimately, hepatocellular carcinoma (17). In the present study, we observed that the levels of the hepatic inflammatory cytokines TNF- $\alpha$, IFN- $\gamma$ and IL-17 were increased at baseline, and increased markedly after OXA chemotherapy in NAFLD mice. The inflammatory cytokines TNF- $\alpha$, IFN- $\gamma$ and IL-17 have been shown to cause hepatocyte injury by triggering a potent cytotoxic immune response and cell death (17-19). Among these factors, TNF- $\alpha$ acts as a pivotal mediator in the progression of acute liver injury. The overproduction of TNF- $\alpha$ through its receptor activates caspase-3, a member of the family of cysteine proteases, which, in turn, triggers hepatocellular necrosis and the apoptotic pathway (18). TNF- $\alpha$-induced ROS generation sustains c-Jun N-terminal kinase (JNK) activation and leads to hepatocyte apoptosis (20). In addition to the rapid increase in the expression of inflammatory cytokines, the NAFLD mice exhibited an aggravation of liver injury and lower survival rate following OXA chemotherapy. Therefore, the findings of the present study demonstrated that the hepatic inflammation already present in NAFLD is further exacerbated by OXA chemotherapy, resulting in more severe liver injury. 
A
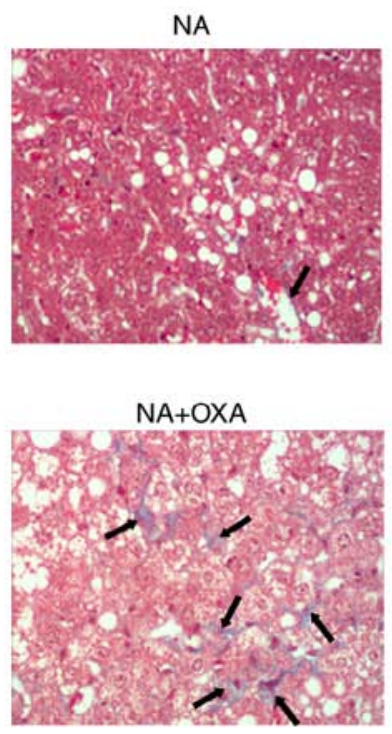
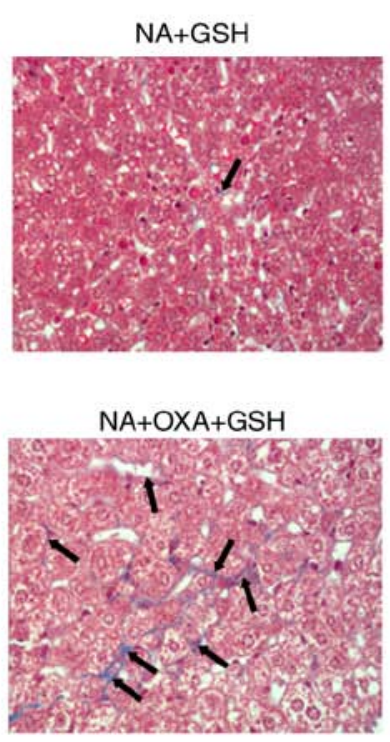

B

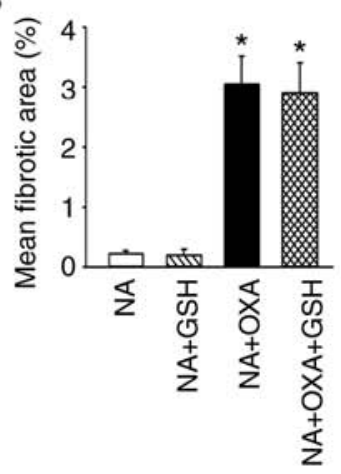

$\mathrm{D}$

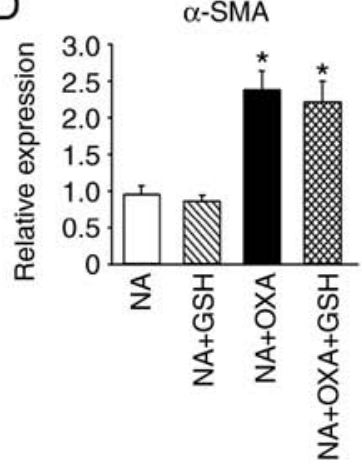

C

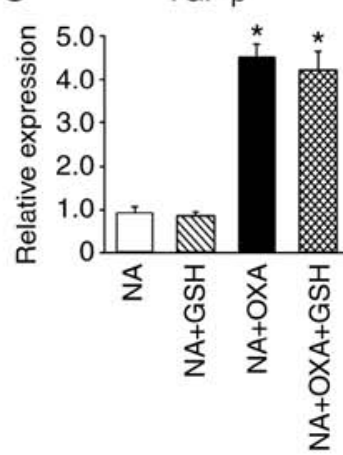

$\mathrm{E}$

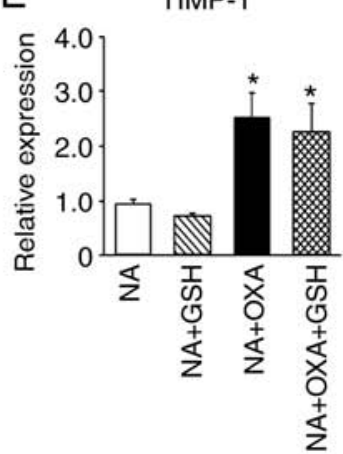

Figure 8. GSH does not attenuate OXA-aggravated liver fibrosis in NAFLD mice. (A) Masson's trichrome staining was performed on liver specimens of NAFLD mice from each group (original magnification, x100). Black arrows indicate areas of collagen deposition. (B) Morphometric analysis of Masson's trichrome staining of the groups in (A). (C-E) mRNA levels of (C) TGF- $\beta$, (D) $\alpha$-SMA and (E) TIMP-1 in liver tissues analyzed by RT-PCR. The results are presented as the means \pm standard deviation from five mice in each group. ${ }^{~} \mathrm{P}<0.05$ compared with the NA group. OXA, oxaliplatin; NAFLD, non-alcoholic fatty liver disease; GSH, reduced glutathione; NA, NAFLD control; TGF, transforming growth factor; SMA, smooth muscle actin; TIMP, tissue inhibitor of metallopeptidase; RT-PCR, reverse transcription-quantitative polymerase chain reaction.

In addition to the observed direct damage to cellular components, recent evidence indicates that oxidative stress plays an important role in the progression of inflammatory disorders. Oxidative stress activates various inflammatory pathways, such as nuclear factor (NF)- $\mathrm{kB}$ and NLRP3, which subsequently induce the expression of the inflammatory cytokines TNF- $\alpha$, IL-1 and TGF- $\beta 1$ (21,22). Excessive ROS generation activates the JNK and caspase pathways, ultimately leading to TNF- $\alpha$-induced cell death (23). Oxidative stress also promotes the migration of inflammatory cells across the endothelial barrier, leading to tissue injury (24). Therefore, it is reasonable to hypothesize that oxidative stress, which is exacerbated by OXA, may contribute to the rapid increase in the production of inflammatory cytokines in NAFLD mice after OXA chemotherapy, further aggravating liver injury. Moreover, the aggravation of hepatic oxidative stress and inflammation after OXA chemotherapy may explain why the risk of liver failure, or even death, in patients with NAFLD is significantly higher compared with that in patients without NAFLD. However, the exact mechanism underlying the interaction between oxidative stress and inflammation after OXA chemotherapy in NAFLD requires further investigation.

The present study also demonstrated that OXA chemotherapy promotes hepatic fibrosis in NAFLD mice, with increased collagen fiber deposition in the liver. OXA has been shown to upregulate the expression of collagen I and TGF- $\beta$ (5). Consistent with this result, we found that TGF- $\beta$ levels increased in both the early and late stages of
OXA-induced acute liver injury in NAFLD mice. TGF- $\beta$ is the most effective cytokine in promoting liver fibrosis, as it can activate hepatic stellate cells (HSCs), promote mass collagen synthesis, and induce gradual deposition of extracellular matrix (ECM) (25). TGF- $\beta$ activates receptor-activated Smads (R-Smads), leading to transcriptional induction of $\alpha$-SMA, the main marker of transdifferentiation of HSCs (26). By upregulating TIMPs to reduce ECM degradation, TGF- $\beta$ further promotes the occurrence and progression of liver fibrosis (25). In the present study, $\alpha$-SMA and TIMP-1 were found to be upregulated during the late stages of OXA-induced acute liver injury. These findings indicated that OXA promotes liver fibrosis in NAFLD mice. Furthermore, ROS are known to promote signaling pathway protein phosphorylation, activation of HSCs, and marked upregulation of TGF- $\beta$ expression (27). Additionally, the inflammatory cytokine IL-17 has been shown to be a profibrotic factor through HSC activation (28). In the present study, a marked increase in the ROS and IL-17A levels in liver tissues was observed following OXA chemotherapy. Therefore, it was hypothesized that the aggravation of OXA chemotherapy-induced oxidative stress and inflammation further promoted the development of liver fibrosis in NAFLD mice.

GSH is the most abundant cellular thiol antioxidant, attaining concentrations in the high millimolar range in the liver (29). GSH can alleviate oxidative stress by serving as a substrate for antioxidative enzymes, including GSH-Px, which converts hydroperoxide into less harmful fatty acids, water 
and GSH disulfide (29). Increased ROS production causes extensive damage to hepatocytes, and ROS can be neutralized by GSH (30). In addition, GSH affects the function of the immune system and regulates cytokine production $(29,30)$. As it is essential to normal liver function, GSH has been extensively used in the clinical setting to suppress liver inflammation $(16,29)$. We previously reported that GSH treatment can significantly inhibit oxidative stress response and acute liver injury induced by OXA in normal mice (7). Accordingly, in the present study, we observed a significant improvement in the cellular redox status, including marked decreases in the levels of ROS, MDA and TNF-1, as well as significantly elevated SOD and GSH-Px activities in the NAFLD mouse liver after OXA chemotherapy, which was accompanied by decreased liver tissue injury. Taken together, these findings suggest that GSH attenuates OXA-induced liver injury in NAFLD mice by inhibiting hepatic oxidative stress and inflammatory cytokine production.

Passive GSH uptake is limited, due to an unfavorable concentration gradient between the plasma and the cytosol (31). Therefore, non-toxic compounds, such as $\gamma$-glutamylcysteine (31) and L-oxothiazolidine carboxylic acid (32), which can increase endogenous intracellular GSH levels and protect against several types of insults, may be more effective in reducing OXA-induced hepatotoxicity, which warrants further investigation.

However, GSH treatment did not completely inhibit OXA chemotherapy-induced liver injury in NAFLD mice, as the ALT and AST levels remained higher compared with those in the NAFLD control group. Additionally, GSH treatment did not increase the survival rate of the NAFLD mice following OXA chemotherapy. These results indicated that GSH treatment does not fully inhibit OXA-induced liver injury. GSH is the most important and ubiquitous antioxidant molecule produced in the liver, where it serves as the principal non-protein thiol involved in antioxidant cell defense (29). Consistently, GSH prevented oxidative liver damage induced by OXA in NAFLD mice, which was confirmed by the significant decrease in the levels of hepatic ROS and MDA following GSH treatment. GSH also reduced the inflammatory cytokine TNF- $\alpha$ level in liver tissues, but did not reduce the levels of IFN- $\gamma$ and IL-17. Therefore, GSH treatment did not completely suppress the OXA-induced hepatic inflammation in NAFLD mice. Furthermore, GSH treatment did not reverse the OXA-induced increase in collagen fiber deposition or the upregulated expression of TGF- $\beta, \alpha$-SMA and TIMP-1 in NAFLD mice, indicating that GSH treatment did not attenuate OXA-aggravated liver fibrosis in NAFLD mice. The pathogenesis of OXA-induced liver injury involves various factors and interactions. It is difficult to completely alleviate liver injury and suppress the development of liver fibrosis using a single hepatoprotective drug, as oxidative stress response, inflammation, fibrosis, thrombosissis and coagulation abnormalities must also be taken into consideration $(5,33)$. Thus, it is crucial to employ a comprehensive therapy scheme to treat OXA-induced liver injury in a background of NAFLD.

Diagnosis of OXA-induced liver injury is mainly based on liver histopathology $(3,5)$. In the present study, the clotting time and serum albumin of NAFLD mice did not differ significantly before and after OXA chemotherapy (data not shown).
Therefore, the liver function tests of biosynthetic capacity may not accurately reflect the hepatoprotective effect of GSH. In this study, the hepatoprotective effect of GSH against OXA-induced liver injury in NAFLD mice was investigated only by liver histopathology and serum ALT and AST levels, without liver function tests of biosynthetic capacity.

In conclusion, the present study demonstrated that OXA induces acute liver injury in NAFLD mice. Moreover, OXA aggravates hepatic oxidative stress in NAFLD mice, as shown by an increase in the ROS and MDA levels and a decrease in the SOD and GSH-Px levels. OXA aggravates hepatic inflammation in NAFLD mice by increasing the expression of the inflammatory cytokines TNF- $\alpha$, IFN- $\gamma$ and IL-17. Furthermore, OXA exacerbates hepatic fibrosis in NAFLD mice, as confirmed by an increase in collagen fiber deposition and TGF- $\beta, \alpha-$ SMA and TIMP-1 levels. Treatment with exogenous GSH can mitigate OXA-induced hepatocyte injury in NAFLD mice by inhibiting hepatic oxidative stress and the production of the pro-inflammatory cytokine TNF- $\alpha$. However, GSH does not attenuate OXA-aggravated liver fibrosis in NAFLD mice. Therefore, these results suggest that GSH may be utilized as a therapeutic agent for the prevention of liver injury caused by OXA treatment in NAFLD; however, GSH does not completely inhibit the OXA-induced liver injury. Therefore, to minimize OXA-induced liver injury in NAFLD, it may be possible to use a concomitant hepatoprotective treatment, whereas all factors that may cause liver damage after OXA chemotherapy should be taken into consideration.

\section{Acknowledgements}

Not applicable.

\section{Funding}

The present study was partially supported by the National Natural Science Foundation of China (grant no. 81460418), the Self-Raised Funds of Guangxi Health Department (grant no. Z2016483), the Guangxi Natural Science Foundation (grant no. 2016GXNSFBA380218), the Guangxi Basic Ability Promotion Project of Middle-aged and Young Teachers in Colleges and Universities (grant no. 2017KY0121), and the Guangxi Key Laboratory of Molecular Medicine in Liver Injury and Repair (grant no. 16-140-46-18).

\section{Availability of data and materials}

The data generated and analyzed during the present study are available from the corresponding authors on reasonable request.

\section{Authors' contributions}

YLu is the first author and performed all steps of the experiments. YLi, XH, SW, JW assisted YLu in conducting the experiments. YLu wrote the manuscript. CY helped in literature survey and study design. All authors contributed toward data analysis, drafting and critically revising the paper, provided final approval of the version to be published, and agree to be accountable for all aspects of the study. 


\section{Ethics approval and consent to participate}

All animal studies were performed according to the guidelines of the Chinese Council on Animal Care and were approved by the Affiliated Tumor Hospital of Guangxi Medical University Committees on Animal Experimentation.

\section{Patient consent for publication}

Not applicable.

\section{Competing interests}

The authors declare that they have no competing interests.

\section{References}

1. Riddell IA: Cisplatin and oxaliplatin: Our current understanding of their actions. Met Ions Life Sci 18, 2018.

2. Formica V,Zaniboni A, Loupakis F and Roselli M: Noninferiority of three months versus six months of oxaliplatin-based adjuvant chemotherapy for resected colon cancer. How should IDEA findings affect clinical practice? Int J Cancer 143: 2342-2350, 2018

3. Rubbia-Brandt L, Audard V, Sartoretti P, Roth AD, Brezault C, Le Charpentier M, Dousset B, Morel P, Soubrane O, Chaussade S, et al: Severe hepatic sinusoidal obstruction associated with oxaliplatin-based chemotherapy in patients with metastatic colorectal cancer. Ann Oncol 15: 460-466, 2004.

4. Duwe G, Knitter S, Pesthy S, Beierle AS, Bahra M, Schmelzle M, Schmuck RB, Lohneis $\mathrm{P}$, Raschzok N, Öllinger R, et al: Hepatotoxicity following systemic therapy for colorectal liver metastases and the impact of chemotherapy-associated liver injury on outcomes after curative liver resection. Eur J Surg Oncol 43: 1668-1681, 2017.

5. Robinson SM, Mann J, Vasilaki A, Mathers J, Burt AD, Oakley F, White SA and Mann DA: Pathogenesis of FOLFOX induced sinusoidal obstruction syndrome in a murine chemotherapy model. J Hepatol 59: 318-326, 2013.

6. Chun YS, Laurent A, Maru D and Vauthey JN: Management of chemotherapy-associated hepatotoxicity in colorectal liver metastases. Lancet Oncol 10: 278-286, 2009.

7. Lin Y, Li Y, Hu X, Liu Z, Chen J, Lu Y, Liu J, Liao S, Zhang Y, Liang $\mathrm{R}$, et al: The hepatoprotective role of reduced glutathione and its underlying mechanism in oxaliplatin-induced acute liver injury. Oncol Lett 15: 2266-2272, 2018.

8. Issa D, Patel V and Sanyal AJ: Future therapy for non-alcoholic fatty liver disease. Liver Int (38 Suppl 1): S56-S63, 2018.

9. Marchesini G, Bugianesi E, Forlani G, Cerrelli F, Lenzi M, Manini R, Natale S, Vanni E, Villanova N, Melchionda N and Rizzetto M: Nonalcoholic fatty liver, steatohepatitis, and the metabolic syndrome. Hepatology 37: 917-923, 2003.

10. Fiorucci S, Biagioli M and Distrutti E: Future trends in the treatment of non-alcoholic steatohepatitis. Pharmacol Res 134: 289-298, 2018

11. Martinez MA, Vuppalanchi R, Fontana RJ, Stolz A, Kleiner DE, Hayashi PH, Gu J, Hoofnagle JH and Chalasani N: Clinical and histologic features of azithromycin-induced liver injury. Clin Gastroenterol Hepatol 13: 369-376.e3, 2015.

12. Lu Y, Wang X, Yan W, Wang H, Wang M, Wu D, Zhu L, Luo X and Ning Q: Liver TCR $\gamma \delta(+)$ CD3(+) CD4(-) CD8(-) T cells contribute to murine hepatitis virus strain 3 -induced hepatic injury through a TNF- $\alpha$-dependent pathway. Mol Immunol 52 229-236, 2012.

13. Masarone M, Rosato V, Dallio M, Gravina AG, Aglitti A, Loguercio C, Federico A and Persico M: Role of oxidative stress in pathophysiology of nonalcoholic fatty liver disease. Oxid Med Cell Longev 2018: 9547613, 2018.

14. Green CJ, Parry SA, Gunn PJ, Ceresa CDL, Rosqvist F, Piché ME and Hodson L: Studying non-alcoholic fatty liver disease: The ins and outs of in vivo, ex vivo and in vitro human models. Horm Mol Biol Clin Investig: Aug 11, 2018 (Epub ahead of print).
15. Koek GH, Liedorp PR and Bast A: The role of oxidative stress in non-alcoholic steatohepatitis. Clin Chim Acta 412: 1297-1305, 2011.

16. Chen Y, Dong H, Thompson DC, Shertzer HG, Nebert DW and Vasiliou V: Glutathione defense mechanism in liver injury: Insights from animal models. Food Chem Toxicol 60: 38-44, 2013

17. Del Campo JA, Gallego P and Grande L: Role of inflammatory response in liver diseases: Therapeutic strategies. World J Hepatol 10: 1-7, 2018.

18. Gao B: Hepatoprotective and anti-inflammatory cytokines in alcoholic liver disease. J Gastroenterol Hepatol 27 (Suppl 2): S89-S93, 2012.

19. Yano A, Higuchi S, Tsuneyama K, Fukami T, Nakajima M and Yokoi T: Involvement of immune-related factors in diclofenac-induced acute liver injury in mice. Toxicology 293: 107-114, 2012.

20. Rutherford A and Chung RT: Acute liver failure: Mechanisms of hepatocyte injury and regeneration. Semin Liver Dis 28: 167-174, 2008.

21. Mittal M, Siddiqui MR, Tran K, Reddy SP and Malik AB: Reactive oxygen species in inflammation and tissue injury. Antioxid Redox Signal 20: 1126-1167, 2014.

22. Zhang X, Zhang JH, Chen XY, Hu QH, Wang MX, Jin R, Zhang QY, Wang W, Wang R, Kang LL, et al: Reactive oxygen species-induced TXNIP drives fructose-mediated hepatic inflammation and lipid accumulation through NLRP3 inflammasome activation. Antioxid Redox Signal 22: 848-870, 2015.

23. Deng Y, Ren X, Yang L, Lin Y and Wu X: A JNK-dependent pathway is required for TNFalpha-induced apoptosis. Cell 115: 61-70, 2003.

24. van Wetering S, van Buul JD, Quik S, Mul FP, Anthony EC, ten Klooster JP, Collard JG and Hordijk PL: Reactive oxygen species mediate Rac-induced loss of cell-cell adhesion in primary human endothelial cells. J Cell Sci 115: 1837-1846, 2002.

25. Fabregat I and Caballero-Diaz D: Transforming growth factor- $\beta$-induced cell plasticity in liver fibrosis and hepatocarcinogenesis. Front Oncol 8: 357, 2018.

26. Kisseleva T and Brenner DA: Role of hepatic stellate cells in fibrogenesis and the reversal of fibrosis. J Gastroenterol Hepatol (22 Suppl 1): S73-S78, 2007.

27. Lin W, Tsai WL, Shao RX, Wu G, Peng LF, Barlow LL, Chung WJ, Zhang L, Zhao H, Jang JY and Chung RT: Hepatitis $\mathrm{C}$ virus regulates transforming growth factor beta1 production through the generation of reactive oxygen species in a nuclear factor kappaB-dependent manner. Gastroenterology 138: 2509-2518, 2518.e1, 2010.

28. Tan Z, Qian X, Jiang R, Liu Q, Wang Y, Chen C, Wang X, Ryffel B and Sun B: IL-17A plays a critical role in the pathogenesis of liver fibrosis through hepatic stellate cell activation. J Immunol 191: 1835-1844, 2013

29. Yuan L and Kaplowitz N: Glutathione in liver diseases and hepatotoxicity. Mol Aspects Med 30: 29-41, 2009.

30. Wang J, Chen Y, Gao N, Wang Y, Tian Y, Wu J, Zhang J, Zhu J, Fan D and An J: Inhibitory effect of glutathione on oxidative liver injury induced by dengue virus serotype 2 infections in mice. PLoS One 8: e55407, 2013.

31. Peter C, Braidy N, Zarka M, Welch J and Bridge W: Therapeutic approaches to modulating glutathione levels as a pharmacological strategy in Alzheimer's disease. Curr Alzheimer Res 12: 298-313, 2015.

32. Gross CL, Giles KC and Smith WJ: L-oxothiazolidine 4-carboxylate pretreatment of isolated human peripheral blood lymphocytes reduces sulfur mustard cytotoxicity. Cell Biol Toxicol 13: 167-173, 1997

33. Zou X, Wang Y, Peng C, Wang B, Niu Z, Li Z and Niu J: Magnesium isoglycyrrhizinate has hepatoprotective effects in an oxaliplatin-induced model of liver injury. Int J Mol Med 42: 2020-2030, 2018.

This work is licensed under a Creative Commons Attribution-NonCommercial-NoDerivatives 4.0 International (CC BY-NC-ND 4.0) License. 